\title{
Poly(ADP-ribose) polymerase (PARP)-based biology and pharmacology for clinical oncotherapy
}

\author{
Eriko Osaki and Shinya Mizuno* \\ Department of Microbiology and Immunology, Osaka University Graduate School of Medicine, Japan
}

\begin{abstract}
PARP1 is a biological sensor for restoring DNA damage, especially at sites of single strand break (SSB). PARP1 inhibition induces an enhancement of SSB, resulting in conversion to double strand break (DSB), genome instability and eventually apoptosis. Such an anti-tumor event by PARP1 inhibitors (PARPi) is selectively induced in cancer cells with a mutation of homologous recombination (HR)-associated molecules, such as BRCA1/2 and ATM (called BRCAness). DNA-damaging chemotherapy and irradiation also sensitize cancer cells to PARPi. Pharmaceutical researchers are now developing PARPi as a novel anti-tumor drug. This review summarizes recent information on PARPi development and its clinical outcomes.
\end{abstract}

\section{Biology of PARP for synthetic lethality induction}

As its name indicated, PARP1 is an enzyme for adding poly(ADPribose) to target proteins (i.e., poly-ADP-ribosylation) [1]. This polymerase plays a central role in DNA repair, especially at SSB sites. If PARP activity is arrested by PARPi, unrepaired SSB is converted to DSB during S-phase of the cell cycle. In response to PARPi-induced DSB, HR system is activated to restore DNA damage through BRCA1/2-based mechanisms [2]. First, DSB is detectable by MRE11A-RAD50-NBS1 complex, leading to a recruitment of DNA checkpoint kinase, ATM. BRCA2 is critical for resecting 5' DNA side, while BRCA2 binds to PALB2 for facilitating RAD51 loading at the DSB site. Under a RAD51loaded condition, homologous double helix is incorporated in the DSB site, thus contributing to DNA repair as a homologous DNA template (i.e., error-free repair). Inversely, PARPi-treated cancer cells undergo "apoptosis" under an HR-deficient condition, including BRCAness, and this is the first model of "synthetic lethality" in experimental cancers, using PARPi $[2,3]$. DNA-damaging therapy, such as chemotherapy, can be a primary option for controlling several types of malignant cancers, and interestingly, DNA-damaging drug sensitizes cancer cells to PARPi. In this process, some chemical agents decrease the expression levels of HR-associated proteins. For instance, a new type of androgen receptor inhibitor, enzalutamide is used for arresting the prostate cancer progression. Notably, enzalutamide decreased the protein levels of BRCA1/2, RAD51 and RAD54 in vitro and in vivo, all of which are necessary for driving HR system, thus mimicking the pharmacological BRCAness [4]. Several lines of evidence indicate that cancer cells become sensitive to PARPi, in combination with irradiation, possibly via HR-impaired (and DSB-enhanced) cascades [5]. The clinical utilities of PARPi are now extended beyond genotypic BRCAness $[1,6]$, suggesting a large market of PARPi for clinical medicine. Based on this background, pharmaceutical companies challenged to develop new types of PARPi, as described below.

\section{Development of PARP inhibitor (PARPi)}

PARP1 is an abundant enzyme for poly-(ADP)-ribosylation of targeted proteins by use of nicotinamide adenine dinucleotide $\left(\mathrm{NAD}^{+}\right)$as a co-factor [1]. There are now several types of chemical compounds to compete with binding of $\mathrm{NAD}^{+}$(a natural ligand of the enzyme) to PARP1/2. Indeed, almost all PARPi have a nicotinamide "pharmacophore" that results in competitive inhibition with $\mathrm{NAD}^{+}$by masking PARP1/2 catalytic domain. BRCA-mutated cancers may be one of the most reasonable indications by PARPi. Now, three types of PARPi are commercially available after FDA approval in the United States [1,7].

\section{Olaparib}

This oral drug is developed by Kudos as an initial code-number, ku-0059436, and later it was licensed by AstraZeneca (Cambridge, UK) as AZD-2281. Olaparib is the first drug to be approved by FDA and EMA in 2014. Pharmacologically, 50\%-inhibitory concentration $\left(\mathrm{IC}_{50}\right)$ of olaparib for PARP1 was $5 \mathrm{nM}$ in vitro, and the time to maximal blood concentration in pharmacokinetics $\left(\mathrm{PK}_{\mathrm{tmax}}\right)$ was 1-3 hours in vivo, with a half-life time $\left(\mathrm{PK}_{\mathrm{t} 1 / 2}\right)$ of 5-6 hours [7]. In a Phase-II trial in 57 patients with recurrent BRCA-mutant ovarian cancer, overall responsive rate (ORR) was $33 \%$ in the high dose cohort (i.e., $400 \mathrm{mg}$ twice a day) [8]. Another Phase-II trial in 63 patients with recurrent high-grade ovarian cancer or triple-negative breast cancer showed a higher ORR in BRCA-mutated group (41\%) than in non-mutated group (24\%) [9]. These studies accelerated the fast approval of olaparib by FDA for treating BRCA-mutated ovarian and breast cancers. Such a beneficial effect of olaparib was also seen in metastatic prostate cancers, especially in BRCA-mutated group, where $14 / 16$ patients (88\%) were well-responded [10]. Based on this result, FDA awarded "breakthrough designation" to develop olaparib for treating the subset of metastatic cancer patients (TOPARP-A trial).

Correspondence to: Shinya Mizuno, Department of Microbiology and Immunology, Osaka University Graduate School of Medicine, Japan, E-mail: mizuno@onbich.med.osaka-u.ac.jp or caepn030@yahoo.co.jp

Key words: BRCAness, DNA damage, homologous recombination, oncotherapy, PARP-trapping effect, synthetic lethality

Received: January 10, 2018; Accepted: January 26, 2018; Published: January 30 2018 


\section{Rucaparib}

AG-014699 or PF-01367338 is a code-number of rucaparib, a PARPi discovered by Newcastle University and Agouron Pharma in 2000, and then developed as an oral drug by Clovis Oncology Inc. Rucaparib produces pharmacological effects at a lower $\mathrm{IC}_{50}$ of $1.4 \mathrm{nM}$ in vitro, and $\mathrm{PK}_{\mathrm{t} 1 / 2}$ is 17-19 hours in vivo [7], suggesting a long-lasting drug. The maximum tolerated dose of rucaparib in the patients with advanced tumors is $600 \mathrm{mg}$ twice a day. Importantly, disease control rate (including complete/partial response and stable disease, $>24$ weeks) was $70 \%$ in germline BRCA-mutant breast cancer patients receiving rucaparib [11]. Rucaparib also obtained FDA breakthrough designation award in 2015. On December 2016, FDA granted fast-inclass approval to rucaparib for treating advanced ovarian cancers with deleterious BRCA mutation (germline or somatic) [12]. Phase-III trials are currently in progress for arresting metastatic prostate cancers with HR-deficiency (NCT02975934; TRITON3) or BRCA-mutated ovarian cancer (NCT02855944; ARIEL4) [7].

\section{Niraparib}

Tesaro developed Niraparib, formerly known as MK-4827, as an orally active small molecule inhibitor of PARP1/2. The value of $\mathrm{IC}_{50}$ is $3.8 \mathrm{nM}$ for PARP1, being similar to that of olaparib, but $\mathrm{PK}_{\mathrm{t} 1 / 2}$ is much longer in niraparib (48-51 hours) than in oraparib (5-6 hours). Phase I monotherapy study has been completed in more than 100 patients with advanced solid tumors, and $300 \mathrm{mg}$ once per day was established as the maximum tolerated dose [13]. Based upon the results of the Phase I/ II study, effectiveness and safety of niraparib are now being evaluated in Phase-II/III trials for targeting ovarian cancer with HR-deficiency (NCT02655016; PRIMA), breast cancer with germline BRCA mutation (NCT 01905592; BRAVO) and metastatic prostate cancer with DNA repair abnormalities (NCT02854436) [7]. In addition to synthetic lethal effect, PARPi may have a direct cytotoxic effect on cancer cells. Actually, trap of PARP1/2 on damaged DNA by PARPi leads to arresting DNA replication and eventually producing cytotoxic effects. Such a PARP-trapping effect was stronger in niraparib-treated cancer cells than olaparib- or rucaparib-treated cells [14]. The trapping effect by niraparib may expand the clinical utility, and future study would shed more light on this speculation.

\section{Veliparib}

In contrast to niraparib, veliparib (ABT-888) is an oral inhibitor that has less trapping activity. Pharmacological information includes: (i) $\mathrm{IC}_{50}$ value is $5.2 \mathrm{nM}$ for PARP1 (and $2.9 \mathrm{nM}$ for PARP2) in vitro; and (ii) $\mathrm{PK}_{\mathrm{tmax}}$ and $\mathrm{PK}_{\mathrm{t} 1 / 2}$ are 3-6 hours and 24 hours in vivo, respectively. In a Phase-I study in patients with BRCA-mutant cancer, maximum tolerated dose was established at $400 \mathrm{mg}$ twice a day and ORR value was $23 \%$ in BRCA-mutant group and $4 \%$ in BRCA-wild type group [15]. Recently, effectiveness of veliparib was evaluated in a Phase-II trials where 50 patients with BRCA-mutant ovarian cancer, fallopian tube cancer or primary peritoneal cancer were enrolled. The ORR was much higher in platinum-sensitive group (35\%) than in platinumresistant group (20\%) [16]. Potential approval of veliparib by FDA in the suppression of cancer progression warrants further attention.

\section{Talazoparib}

BMN-673 is a code-number of PARPi candidate, developed by Medivation. Talazoparib has the most potent PARP-trapping activity among FDA-approved drugs and another candidate drug. Of note, $\mathrm{IC}_{50}$ of $\mathrm{BMN}-673$ is $0.58 \mathrm{nM}$ (i.e., $11 \%$ of that in olaparib) and $\mathrm{PK}_{\mathrm{t} 1 / 2}$ is 48-hours (i.e., 8-fold longer than in olaparib) [7]. In the Phase-I clinical trial for patients with advanced solid tumor (NCT01286987), maximum tolerated dose was $1 \mathrm{mg} /$ day, and this result may be linked with the lower $\mathrm{IC}_{50}$ and longer $\mathrm{PK}_{\mathrm{t} 1 / 2}$. Of interest, ORR in this regimen was $50 \%$ in BRCA-mutant breast cancer, $42 \%$ in BRCA-mutant ovarian cancer and $20 \%$ in pancreatic cancer [17]. The Phase-III trial is underway for patients with metastatic germline BRCA-mutant breast cancer (NCT01945775; EMBRACA) [7].

\section{Summary and perspective}

In this article, we discussed the possible biological mechanisms by which PARPi produces cytotoxic effects specifically in cancer cells with HR-deficiency. Principally, synthetic lethal effect should be undetectable in normal cells, because HR system is well-preserved, hence suggesting a selective toxic effect on cancer cells, but not normal cells. So far, several types of PARPi have been developed, according to the competitive activity with $\mathrm{NAD}^{+}$. Needless to say, olaparib (ku-0059436 or AZD2281) is a leading drug for development of PARPi. Olaparib is the first drug to provide a proof-of-concept for synthetic lethality-based oncotherapy, especially under germline or somatic mutations of BRCAness-associated genes [8,9]. Niraprib and talazoprib is also promising for oncotherapy, possibly via a dual cascade of synthetic lethal and PARP-trapping effects $[7,18]$.

Long-term application of PARPi likely produces resistance to PARPi in cancer cells, possibly via activation of alternative pathway for restoring HR-deficiency. Basic research on PARPi resistance is significant for understanding of biological principle and pharmacological development as well. PARPi can be a biological probe to identify a compensated molecule(s), such as ATR, an alternative kinase responsible for restoring DNA damage. BRCAness-related mutant genes may become functional through re-acquiring wildtypelike amino acid sequence. A recent study described that growth factor-receptor system (such as HGF and c-Met axis) may critical for producing PARPi resistance [19].

Another interest is to develop a new type of PARPi with a different active site to overcome PARPi resistance. As repeated, the majority of PARPi used in clinics have been developed as $\mathrm{NAD}^{+}$competitor. Since classical PARPi displays structural similarities to nucleotides, they may obstruct the function of other $\mathrm{NAD}^{+}$-utilizing enzymes, such as IMPDH. To circumvent this problem, Thomas et al. screened the novel inhibitors that block PARP1 activity in cancer cells, based on an ability to block PARP1-histone4 binding. As a result, several lines of histon4-targeted PARPi blocked PARP activity in cancer cells with greater efficacy and potency than classical PARPi. Among them, one chemical compound (5F02) arrested the progression of human cancer in a nude mouse model [20]. The reduction reaction by IMPDH was inhibited by Olaparib, but not 5F02, suggesting a specific targeting effect by new PARPi. Thus, non-NAD ${ }^{+}$type PARPi (i.e., histon4binding motif-masking PARPi) may also be practical for oncotherapy, especially for releasing resistance to classical PARPi. Pharmaceutical development of PARPi will open up a new avenue for advance in genome biology and cancer medicine.

\section{References}

1. Bitler BG, Watson ZL, Wheeler LJ, Behbakht K (2017) PARP inhibitors: Clinical utility and possibilities of overcoming resistance. Gynecol Oncol 147: 695-704 [Crossref]

2. Lord CJ, Ashworth A (2016) BRCAness revisited. Nat Rev Cancer 16: 110-120. [Crossref] 
3. Bryant HE, Schultz N, Thomas HD, Parker KM, Flower D, et al. (2005) Specific killing of BRCA2-deficient tumours with inhibitors of poly(ADP-ribose) polymerase. Nature 434: 913-917. [Crossref]

4. Li L, Karanika S, Yang G, Wang J, Park S, et al. (2017) Androgen receptor inhibitorinduced "BRCAness" and PARP inhibition are synthetically lethal for castrationresistant prostate cancer. Sci Signal 10: eaam7479. [Crossref]

5. Lester A, Rapkins R, Nixdorf S, Khasraw M, McDonald K, et al. (2017) Combining PARP inhibitors with radiation therapy for the treatment of glioblastoma: Is PTEN predictive of response? Clin Transl Oncol 19: 273-278. [Crossref]

6. Lee JM, Ledermann JA, Kohn EC (2014) PARP Inhibitors for BRCA1/2 mutationassociated and BRCA-like malignancies. Ann Oncol 25: 32-40. [Crossref]

7. Ferrara R, Simionato F, Ciccarese C, Grego E, Cingarlini S, et al. (2018) The development of PARP as a successful target for cancer therapy. Expert Rev Anticancer Ther 18: 161-175. [Crossref].

8. Audeh MW, Carmichael J, Penson RT, Friedlander M, Powell B, et al. (2010) Oral poly(ADPribose) polymerase inhibitor olaparib in patients with BRCA1 or BRCA2 mutations and recurrent ovarian cancer: a proof-of-concept trial. Lancet 376: 245-251. [Crossref]

9. Gelmon KA, Tischkowitz M, Mackay H, Swenerton K, Robidoux A, et al. (2011) Olaparib in patients with recurrent high-grade serous or poorly differentiated ovarian carcinoma or triple-negative breast cancer: a phase 2, multicentre, open-label, nonrandomised study. Lancet Oncol 12: 852-861. [Crossref]

10. Mateo J, Carreira S, Sandhu S, Miranda S, Mossop H, et al. (2015) DNA-repair defects and olaparib in metastatic prostate cancer. $N$ Engl J Med 373: 1697-1708. [Crossref]

11. Kristeleit RS, Burris HA, LoRusso P, Patel MR, Asghar US, et al. (2014) Phase 1/2 study of oral rucaparib: Final phase 1 results. J Clin Oncol 32(supple): abstr5513.

12. Konecny GE, Kristeleit RS (2016) PARP inhibitors for BRCA1/2-mutated and sporadic ovarian cancer: current practice and future directions. $B r$ J Cancer 115: 1157-1173. [Crossref]
13. Sandhu SK, Schelman WR, Wilding G, Moreno V, Baird RD, et al. (2013) The poly(ADP-ribose) polymerase inhibitor niraparib (MK4827) in BRCA mutation carriers and patients with sporadic cancer: a phase 1 dose-escalation trial. Lancet Oncol 14: 882-892. [Crossref]

14. Murai J, Huang SY, Das BB, Renaud A, Zhang Y, et al. (2012) Trapping of PARP1 and PARP2 by clinical PARP inhibitors. Cancer Res 72: 5588-5599. [Crossref]

15. Puhalla S, Beumer JH, Pahula S, Appleman J, Tawbl AH, et al. (2014) Final results of phase-I study of single-agent veraprib (V) in patients (pts) with either BRCA1/2mutant cancer $(\mathrm{BRCA}+)$, platinum-refractory ovarian, or basal-like breast cancer (BRCA-wt). J Clin Oncol 32(suppl): abstr2570.

16. Coleman RL, Sill MW, Bell-McGuinn K, Aghajanian C, Gray HJ, et al. (2015) A phase II evaluation of the potent, highly selective PARP inhibitor veliparib in the treatment of persistent or recurrent epithelial ovarian, fallopian tube, or primary peritoneal cancer in patients who carry a germline BRCA1 or BRCA2 mutation - An NRG Oncology/ Gynecologic Oncology Group study. Gynecol Oncol 137: 386-391.

17. de Bono J, Ramanathan RK, Mina L, Chugh R, Glaspy J, et al. (2017) Phase I, doseescalation, two-part trial of the PARP Inhibitor Talazoparib in patients with advanced germline BRCA1/2 mutations and selected sporadic cancers. Cancer Discovery 7: 620-629.

18. Ohmoto A, Yachida S (2017) Current status of poly(ADP-ribose) polymerase inhibitors and future directions. Onco Targets Ther 10: 5195-5208.

19. Du Y, Yamaguchi H, Wei Y, Hsu JL, Wang HL, et al. (2016) Blocking c-Met-mediated PARP1 phosphorylation enhances anti-tumor effects of PARP inhibitors. Nature Med 22: $194-201$.

20. Thomas C1, Ji Y, Lodhi N, Kotova E, Pinnola AD, et al. (2016) Non-NAD-like poly(ADP-Ribose) polymerase-1 inhibitors effectively eliminate cancer in vivo. EBioMedicine 13: 90-98.

Copyright: (C2018 Osaki E. This is an open-access article distributed under the terms of the Creative Commons Attribution License, which permits unrestricted use, distribution, and reproduction in any medium, provided the original author and source are credited. 